\title{
Correspondence
}

\section{Renin and aldosterone response in human newborns to acute blood volume change}

Sir,

We read with interest the report of Dillon and associates (Archives, 1978, 53, 461) on this subject. In a paper to be submitted to Rivista Italiana Pediatria we studied plasma renin activity (PRA) in 13 newborn infants undergoing exchange transfusion with ACD stored blood for hyperbilirubinaemia of various aetiology during the first 6 days of life. Exchange transfusions were such that the infants were kept normovolaemic. Weights ranged from 2400 to $3810 \mathrm{~g}$, and gestational ages from 37 to 41 weeks. PRA and haematocrit were determined on umbilical vein samples before, midway, and at the end of the procedure, and from the transfused blood. The amount of blood administered ranged from 63.7 to $155.8 \mathrm{ml} / \mathrm{kg}$, and the exchange rate from 0.964 to 5.288 $\mathrm{ml} / \mathrm{kg}$ per $\mathrm{min}$.

Statistical analysis of data was performed by a step-wise multiple regression program (UNIVAC 90/30), taking into account as dependent variable the average rate of renin production during the period of exchange transfusion and as independent variables the weight, postnatal age, basal PRA of the newborn, donor's PRA, and exchange rate. Allowing for the dilution caused by the donor's blood, hyperproduction of renin occurred in all cases. All these variables influenced, in various directions, the rate of renin release but the rate of exchange appears to be the most important factor in the activation of the renin-angiotensin system (multiple correlation coefficient $=0.774 ;$ F-value for analysis of variance $=16.404$; standard error of estimate $=0.078 ; t=4.050 ; P<0.01$ )

G. F. Spennati, S. Placidi, B. Persichetti, and F. De Matteis Cattedra di Clinica Pediatrica, Ospedale San Salvatore, 67100 L'Aquila, Italy

\section{Familial neurodegenerative disorder associated with raised urinary VMA} Sir,

The belief held by Young and Hosking (Archives, 1978, 53,682 ) that 'disturbances of catecholamine excretion have not been known to occur in children with a progressive neurological disorder or other neurological insult' is not true. Excessive urinary excretion of 4-hydroxy-3-methoxymandelic acid (HMMA) has been demonstrated in hydrocephalic infants with spina bifida
(Barson and Hodge, 1971). It was felt from this study that a raised urinary HMMA reflected a persistently raised intracranial pressure, since it was not a feature of spina bifida alone or in cases where hydrocephalus was well controlled by a valve. It is likely that increased excretion of catecholamines is a nonspecific consequence of degenerating nerve tissue, whether this is in turn due to increased intracranial pressure or to an intrinsic neurological disorder.

\section{Reference}

Barson, A. J., and Hodge, J. S. (1971). Urinary 4-hydroxy-3methoxymandelic acid (HMMA) excretion in infants with spina bifida. Developmental Medicine and Child Neurology, 13, Supplement 25, 19-26.

\section{A. J. BARSON \\ Department of Pathology, St Mary's Hospital, Whitworth Park, Manchester M13 OJH}

\section{Dr Hosking comments:}

We are grateful to Dr Barson for drawing our attention to his important paper. It was remiss of us not to be aware of the data in that publication.

The raised levels of urinary HMMA seen in Drs Barson and Hodge's papers are most likely nonspecific as Dr Barson suggests. Similar increases are seen from time to time in a number of 'stress' situations. However, in our patient there was not only biochemical evidence of impaired catecholamine metabolism or excretion, but also clinical features apparently related directly to this abnormality. It is for this reason we suspect our patient's disordered metabolism was rather more specific.

GWILYM Hosking The Ryegate Centre, The Children's Hospital, Sheffield S10 5DD

\section{Peripheral pulmonary artery stenosis}

Sir,

The observation by Salisbury and Keeling (Archives, $1978,53,428$ ) of polypoid lesions in the peripheral pulmonary arteries of an infant, recalls a controversy that was resolved over 20 years ago. Originally described in systemic arteries by Bucciante in 1945 and later by many others, these structures became known as 'pedunculated nodules' or 'polypoid cushions' and were interpreted as flow regulation mechanisms. Later studies 